\title{
Specific heat spectra of non-interacting fermions in a quasiperiodic ladder sequence
}

\author{
D.A. Moreira, E.L. Albuquerque*, D.H.A.L. Anselmo \\ Departamento de Física, Universidade Federal do Rio Grande do Norte, 59072-970 Natal-RN, Brazil
}

\section{A R T I C L E I N F O}

\section{Article history:}

Received 17 March 2008

Received in revised form 19 May 2008

Accepted 6 June 2008

Available online 11 June 2008

Communicated by R. Wu

\section{PACS:}

05.30.Fk

05.45.Df

61.43.Hv

$65.60 .+a$

Keywords:

Specific heat

Quasi-crystals

Fractal behavior

Non-interacting fermions

DNA molecule model

\begin{abstract}
A B S T R A C T
We compute the specific heat spectra of non-interacting fermions whose energy spectrum was obtained from a quasiperiodic ladder sequence (Fibonacci and Rudin-Shapiro type), mimicking a DNA molecule model. The specific heat is calculated from their underlying multi-fractal energy spectrum, considering several values of energy densities. Comparisons are made with a real DNA sequence, namely the human chromosome 22 (Ch22).
\end{abstract}

(C) 2008 Elsevier B.V. All rights reserved.
The DNA molecule is often described as a one-dimensional random chain, being defined as a sequence of four possible nucleotides which shapes the structure of the amino acids to form proteins. Its sequence can be considered as a symbolic arrangement of a four Letter alphabet, namely guanine $(G)$, adenine $(A)$, cytosine $(C)$ and thymine $(T)$, and nothing prevents that the DNA chain can be grown following quasiperiodic sequences.

One of the most studied models of a one-dimensional quasiperiodic structure is the Fibonacci lattice, which exhibits a critical behavior of localization of the eigenstates independent of the two values taken by the substitution potential. The criticality of the localization is revealed by a singular continuous energy spectrum consisting of a Cantor set of zero Lebesgue measure for the Fibonacci Hamiltonian. Another one is the Rudin-Shapiro sequence, which holds a unique position inasmuch as its correlation measure is absolutely continuous, such as for random sequences. Based on this feature one would expect the Rudin-Shapiro lattice to have properties close to that of a random system, especially since its correlation measure has a uniform density. Besides, the former (Fibonacci) shows many robust transmission energies in the spec-

\footnotetext{
* Corresponding author. Tel.: +55 84 32153793; fax: +55 8432153791.

E-mail address: eudenilson@dfte.ufrn.br (E.L. Albuquerque).
}

trum, whereas the latter (Rudin-Shapiro) shows weak transmission ability with increasing sequence length (for a review of the physical properties of these quasiperiodic structures, see $[1,2]$ ).

Unlike proteins, a $\pi$-stacked array of DNA base pairs made up from these nucleotides can provide the way to promote long range charge migration, which in turn gives important clues to mechanisms and biological functions of transport $[3,4]$. Furthermore, the characterization of the long-range correlations in DNA sequences have proved to be a difficult task, mainly due to its mosaic-like structure consisted of patches with distinct nucleotide compositions [5], giving rise to local biasing (trends) in their composition, and in turn to spurious effects when analyzed through numerical methods [6]. Besides, the charge transfer efficiency varies for different substitutional sequences, with many of them presenting electronic delocalization [7]. Nevertheless, the mechanistic description of charge transfer in DNA is not comprehensive yet and a complete and clear description of the electronic properties inside the DNA base stack is still lacking. There are many mechanistic details which are being addressed currently or need to be explored in the future. The basic problems of the formation of a complete mechanistic picture are the significant structural difference of the investigated DNA systems as well as the different ways of interpretation. Hence, DNA represents a very special medium in terms of energy and charge transfer processes [8] 
Many interesting theoretical results concerning the electronic properties of one-dimensional chains, including charge transfer mechanism in DNA, have been obtained by using the Schrödinger equation in the tight-binding approximation, i.e.:

$t\left(\psi_{n+1}+\psi_{n-1}\right)=\left(E-\epsilon_{n}\right) \psi_{n}$,

where $\epsilon_{n}$ is the single energy at the orbital $\psi_{n}$, whereas $t$ is the common first-neighbor electronic overlap (hopping amplitude). A considerable amount of work has been devoted to the study of this equation, for both random and quasiperiodic sequences of the potential $V_{n}$. The main achievements were:

(a) if the potentials $V_{n}$ are independent random variables, the system exhibits Anderson model of localization, i.e., all the eigenstates are exponentially localized, and the energy of the spectrum itself is always a regular object, with at most a finite number of bands $[9,10]$. In the case of a binary potential distribution, the spectrum has one or two bands.

(b) If the potentials $V_{n}$ are a binary sequence arranged in a pure Fibonacci or generalized Fibonacci way, the energy spectrum is a Cantor set of zero (Lebesgue) measure, i.e., there is an infinite number of gaps, and the total bandwidth vanishes. Specifically for the pure Fibonacci case, the eigenstates are neither extended nor localized, but exhibit an intermediate behavior. For the generalized Fibonacci case, the eigenstates are extended [11,12]. In higher-dimensional cases, the energy spectra can be band-like with finite measure, fractal-like with zeroband-width, or a mixture of partly band-like and partly fractal-like character [13].

Simplified fractals based on the triadic Cantor set [14,15], as well as on the critical attractor of the logistic and circle maps [16, 17], have been used recently to model the energy spectrum of quasiperiodic systems. More sophisticated methods, based on the Schrödinger equation, were also proposed, looking for connections with the quasiperiodic aspects of these spectra (scaling laws, fractal dimension, etc.) as well as for some kind of common behavior in the specific heat spectra. It has been shown, among other things, that the average low-temperature specific heat is intimately connected with some underlying fractal dimension characterizing the energy spectrum [18].

It is the aim of the present work to contribute to this subject considering the energy spectra of a quasiperiodic ladder sequences (Fibonacci and Rudin-Shapiro type), mimicking a DNA molecule. Taking them as the energy spectrum of a single fermionic particle, we will compute the specific heat of a system of non-interacting fermions and discuss its features in connection with the scale invariance character of their underlying multi-fractal energy spectrum. Comparisons are then made with a real DNA sequence, namely the human chromosome 22 (Ch22).

Our Hamiltonian is the effective tight-binding model describing one electron moving in a chain with a single orbital per site and nearest-neighbor interactions, as given by Eq. (1).

The quasiperiodic Fibonacci (FB) sequence is constructed starting from a $G$ (guanine) nucleotide as seed and following the inflation rule $G \rightarrow G C, C \rightarrow G$. On the other hand, starting, as in the Fibonacci case, from a $G$ (guanine) nucleotide as seed, the quasiperiodic Rudin-Shapiro (RS) can be built through the inflation rules $G \rightarrow G C, C \rightarrow G A, A \rightarrow T C$, and $T \rightarrow T A$. For both quasiperiodic chains, we consider that the energies $\epsilon_{n}$ take the values $\epsilon_{G}$, $\epsilon_{A}, \epsilon_{C}$, and $\epsilon_{T}$ as in the DNA genomic sequence, with the same numerical values.

For the DNA sequence of the first sequenced human chromosome 22 (Ch 22), entitled $N T_{011520}$, the numbers of letters of this sequence is about $3.4 \times 10^{6}$ nucleotides. This sequence was retrieved from the internet page of the National Center of Biotech- nology Information. The energies $\epsilon_{n}$ are chosen from the ionization potential of the respective bases [19], i.e., $\epsilon_{A}=8.24, \epsilon_{T}=9.14$, $\epsilon_{C}=8.87$, and $\epsilon_{G}=7.75$, all units in $\mathrm{eV}$, representing the adenine, guanine, thymine, and cytosine molecules. The hopping term $t$ among the bases was taken equal to $1 \mathrm{eV}$. This specific value is chosen because theoretical ab initio simulations yield values between 0.4 and $1 \mathrm{eV}[20,21]$.

The multifractal energy spectrum of the FB and RS sequences, for both single- and double-strands DNA-like sequences, was obtained by us in previous works [22,23], and it will be taken into account to determine the specific heat spectra. Since the spin degree will not be considered in this work, each occupied quantum state can support only one particle. According to the Fermi-Dirac statistics, the average occupation number of each state is given by

$$
\left\langle n_{i}\right\rangle=\frac{1}{1+\exp \left[\beta\left(\varepsilon_{i}-\mu\right)\right]},
$$

where $\beta=1 / T$ (we are using units of $k_{B}=1$ ) and $\mu$ is the chemical potential, which can be computed as a function of temperature and band filling from

$N_{e}=\sum_{i=1}^{N}\left\langle n_{i}\right\rangle$,

from which $\mu\left(N_{e} / N, T\right)$ can be extracted by numerical methods.

The average internal energy is obtained from

$U\left(N_{e} / N, T\right)=\sum_{i=1}^{N} \varepsilon_{i}\left\langle n_{i}\right\rangle$,

where the temperature dependence of the chemical potential $\mu$ is explicitly taken into account.

We compute the specific heat at constant volume by differentiating $U\left(N_{e} / N, T\right)$ with respect to the temperature $T$, i.e., $C_{V}=d U\left(N_{e} / N, T\right) / d T$. It is then straightforward to calculate the fermionic specific heat yielding

$$
\begin{aligned}
C_{V}= & \frac{1}{4 T^{2}}\left[\sum_{i} \varepsilon_{i}^{2} \cosh ^{-2}\left[\left(\varepsilon_{i}-\mu\right) / 2 T\right]\right. \\
& \left.-\frac{\left[\sum_{i} \varepsilon_{i} \cosh ^{-2}\left[\left(\varepsilon_{i}-\mu\right) / 2 T\right]\right]^{2}}{\sum_{i} \cosh ^{-2}\left[\left(\varepsilon_{i}-\mu\right) / 2 T\right]}\right] .
\end{aligned}
$$

Fig. 1(a) shows a log-log plot of the electronic specific heat spectra at constant volume (in units of $N_{e}$ ) versus the temperature $T$ for the 14th generation of the Fibonacci DNA chain, corresponding to $n=610$ nucleotides. Several values of the band fillings $N_{e} / N$ are considered, and indicated in the figure. For the high temperature limit $(T \rightarrow \infty)$, the specific heat for all cases converges and decays with $T^{-2}$. As the temperature decreases, the specific heat increases up to a maximum value, the corresponding temperature for this maximum value depends on the number of band fillings $N_{e} / N$, although one can see a clear tendency for a common temperature value as $N_{e} / N$ increases, and this value is independent of the occupation number ratio. After the maximum value, the specific heat falls into the low temperature region and starts, due to the fractality of the energy spectrum, a complex pattern of logperiodic oscillations which signals the discrete scale invariance of the spectrum at the vicinity of the Fermi energy. These oscillations occur around a linear trend (in $\log -\log$ scale), whose power-law behavior is $C_{v} \propto T^{\phi_{\mathrm{FB}}}$, with $\phi_{\mathrm{FB}}=0.7385$, lasting until the temperature reaches a value around $10^{-3}$. At this point, a phase transition (in the sense of a oscillatory regime) occurs, and the specific heat falls again linearly with $T$. The inset of this figure considers the 15th generation of the Fibonacci DNA chain, corresponding to $n=987$ nucleotides. From there we can see a larger number of oscillations of the specific heat for low $T$ (in general the number of 


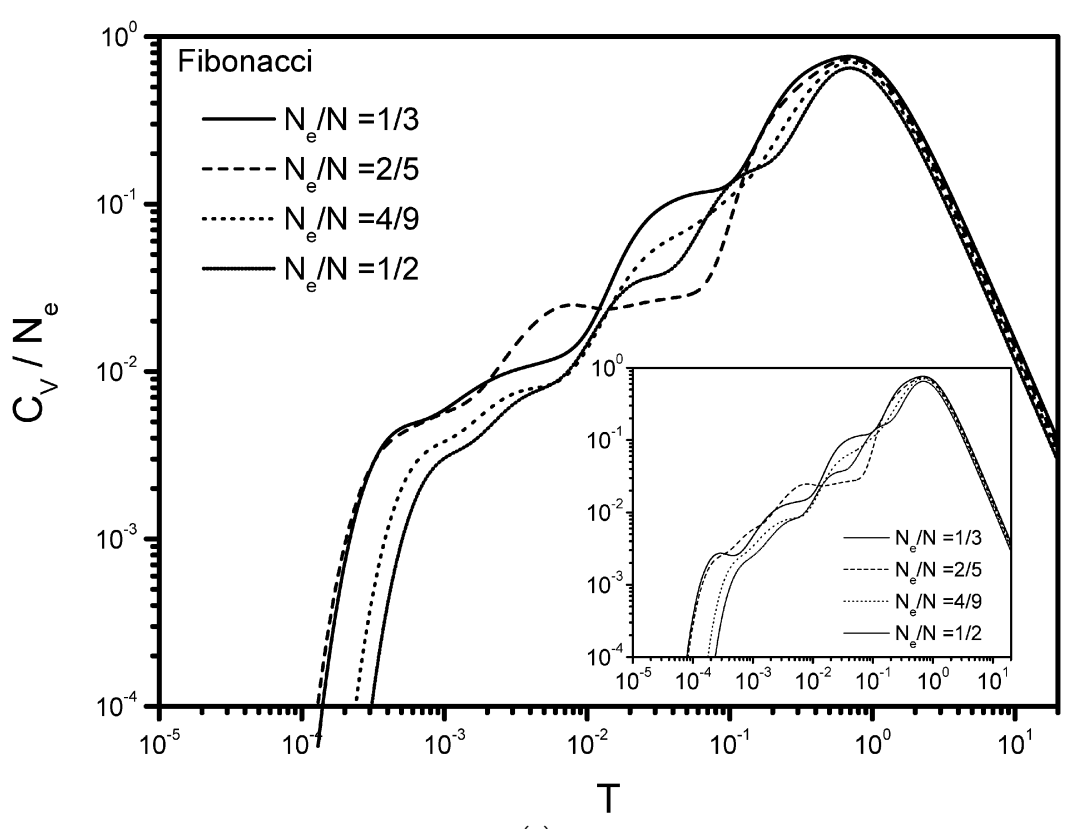

(a)

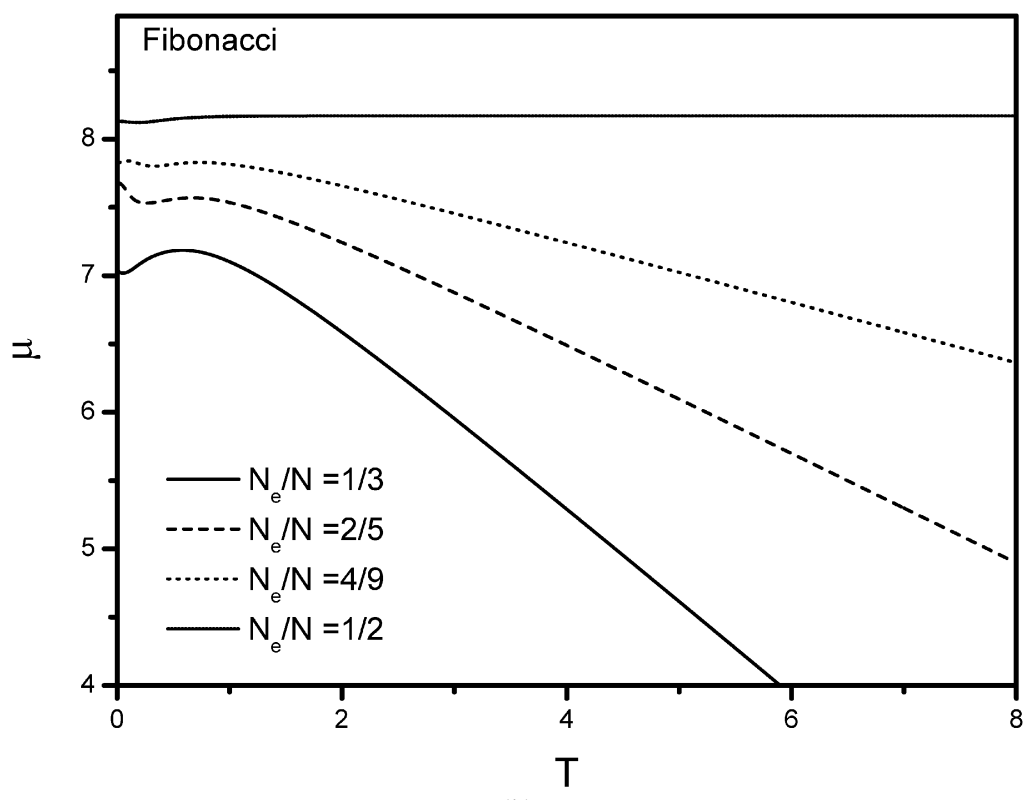

(b)

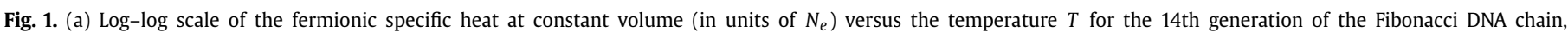

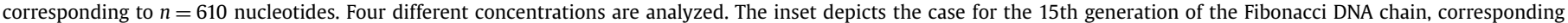
to $n=987$ nucleotides. (b) Chemical potential $\mu$ versus the temperature $T$.

oscillations of the specific heat for fractal spectra increases with the order of the generation of the fractal). Besides, the oscillatory regime disappears at a lower temperature, when compared to the 14th generation of the Fibonacci DNA chain.

In Fig. 1(b), the profile of the chemical potential $\mu\left(T, N_{e} / N\right)$ against the temperature $T$ is presented, for a Fibonacci DNA chain, considering the occupation ratios $N_{e} / N=1 / 2,4 / 9,2 / 5$ and $1 / 3$. For lower values of $T$, there is a transient period, on which the chemical potential reaches a maximum value, and then starts to decrease (in all cases but $N_{e} / N=0.5$ ) linearly, as the ratio $N_{e} / N$ decreases. For $N_{e} / N=0.5$, the chemical potential has a constant value. This is an expected feature, since the chemical potential is a measure of the energy per particle, for a given entropy.

A similar scenario appears when one considers the other quasiperiodic structure studied, modeling the DNA molecule by the Rudin-Shapiro sequence, whose log-log plot of the specific heat at constant volume (in units of $N_{e}$ ) is depicted in Fig. 2(a), considering its 10th generation (which means 512 nucleotides). Its profile is very similar to that of Fig. 1, when one considern the same band fillings $N_{e} / N$. There is a transient region where the $C_{V}$ oscillates non-harmonically around an inclined straight line, $C_{v} \propto T^{\phi_{\mathrm{RS}}}$, with $\phi_{\mathrm{RS}}=1.01$, and then suddenly if falls to zero, linearly with $T$. However, now this decrease depends more strongly on the band fillings $N_{e} / N$ considered. The inset shows the case for the RudinShapiro's 11th generation, corresponding to $n=1024$ nucleotides. The chemical potential for this sequence is shown in Fig. 2(b), with qualitative behavior similar to the FB case, but now presenting a shift for the $\mu$ values. In other words, the energy per particle is higher when one consider the DNA-like chain modeled by the RS sequence.

The above studied DNA-like chains give us reasonable clues on how the energy distributions would behave in a real DNA chain. 


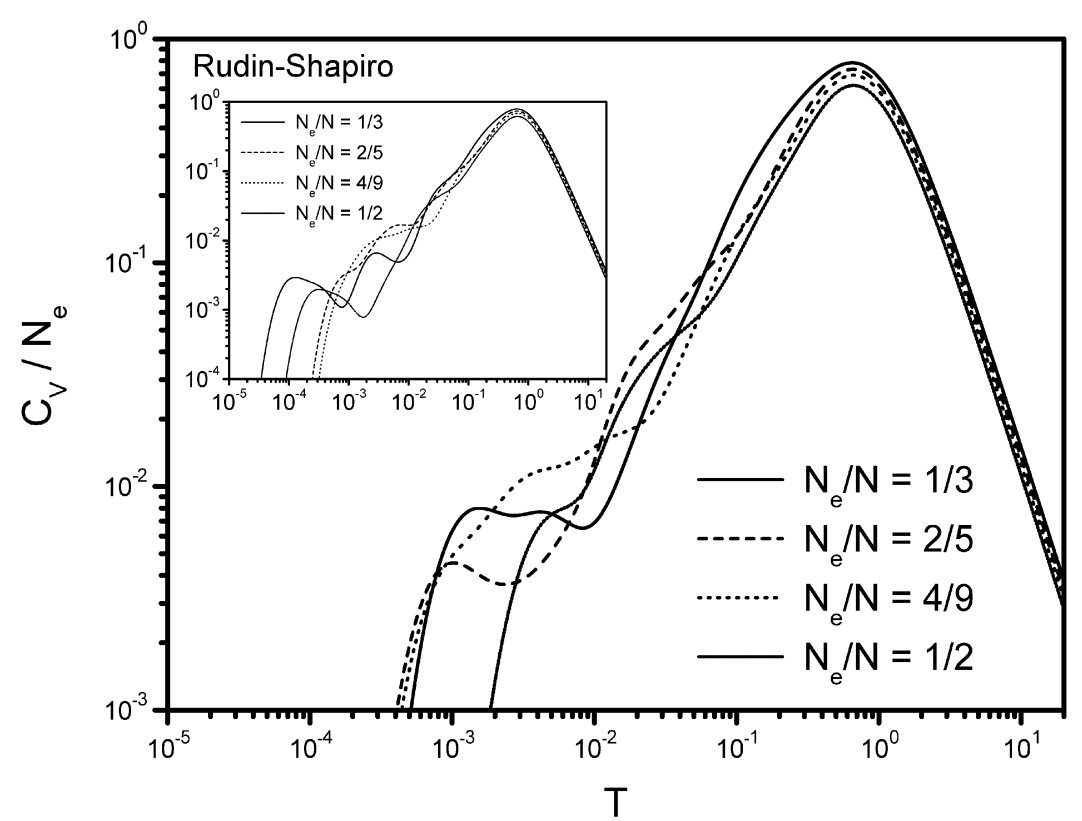

(a)

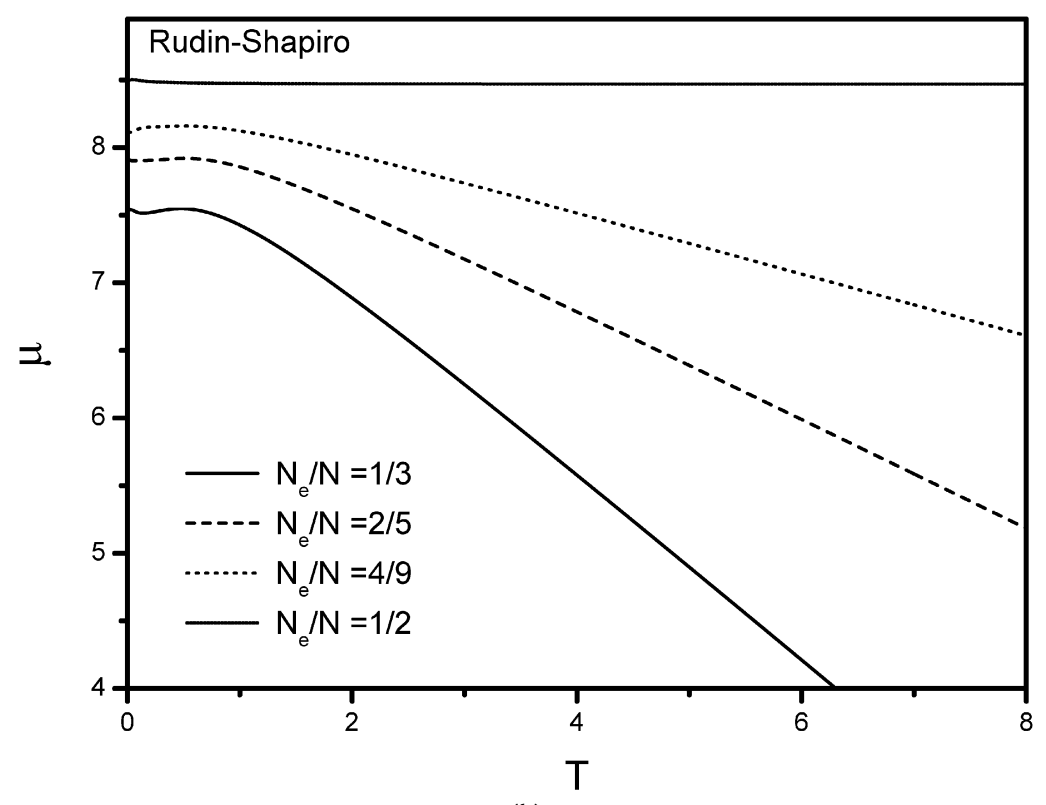

(b)

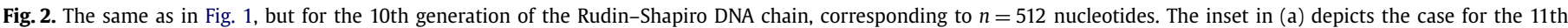
generation of the Rudin-Shapiro DNA chain, corresponding to $n=1024$ nucleotides.

Indeed, purely theoretical efforts have been made [24,25] to understand the underlying thermodynamics of fractal spectra and quasiperiodic systems. However, this work would not be complete if we did not compare our results with a real system. For this purpose, the log-log plot of the specific heat at constant volume (also in units of $N_{e}$ ) for the Ch22 chromosome, considering $n=512$ nucleotides, analyzed through a Fermi-Dirac statistics, is depicted in Fig. 3(a). Again, the specific heat falls to zero when $T \rightarrow \infty$, but now in a slightly higher ratio. Also, after the maximum value of $C_{V}$ is reached, in the low temperature region the specific heat falls roughly linearly with $T$, and at $T=0.5 \times 10^{-2}$, it falls more rapidly with $T$. Note that in this case there is less oscillations when $T \rightarrow 0$ when compared to the quasiperiodic structures. Probably this is due to the fact that, in contrast to a real fractal, human chromosomes present a common compositional structure with two characteristic scales, the large one corresponding to long, homogeneous DNA segments (the isochores), and the other one to small and medium scale genomic elements [26]. The inset presents the case for the human chromosome Ch22 with 1024 nucleotides.

The chemical potential, depicted in Fig. 3(b), resembles strongly the RS one, which means that the energy distribution per particle is very similar in these two cases. This qualitative resemblance is an indication that a real DNA chain can, at least in principle, be modeled through substitutional sequences, like FB and RS. Also, the lack of an oscillatory behavior around a medium value (the spectral or fractal dimension of the system), a common feature presented in previous works, clearly indicates that the statistics considered (namely, the Fermi-Dirac one), which forbids more than one particle per state (excluding the spin), plays a decisive role on the collective behavior of electrons propagating in real and modeled DNA chains.

In conclusion, we have presented in this Letter a theoretical model to study the electrons' specific heat spectra and chemical 


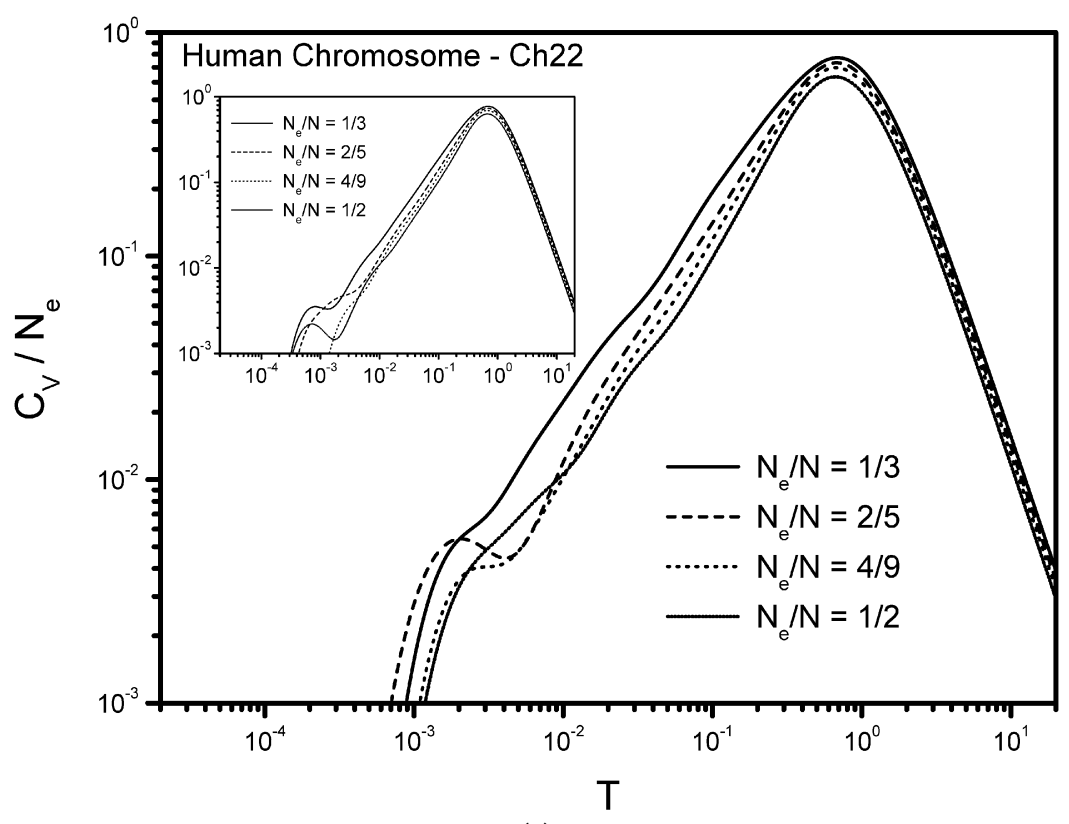

(a)

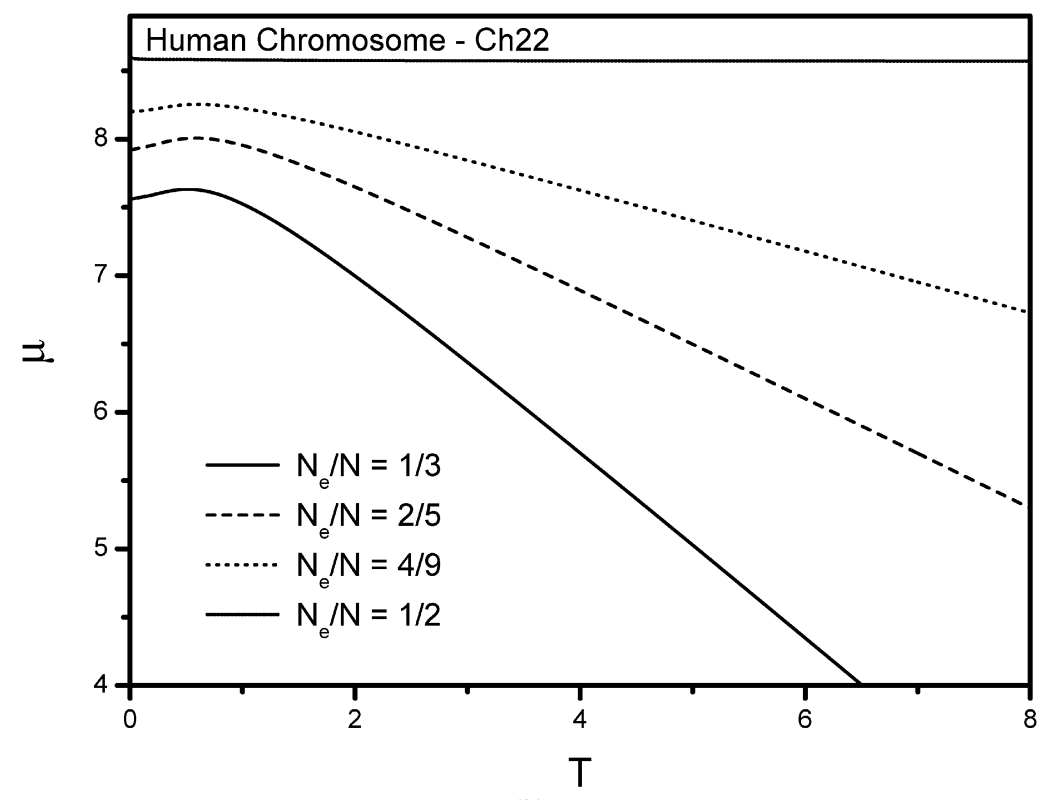

(b)

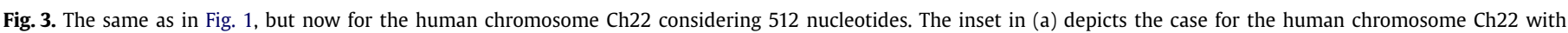
1024 nucleotides.

potential of single strand DNA molecules, made up from the nucleotides $G, C, A$, and $T$, arranged to form two artificial sequences, the Fibonacci and Rudin-Shapiro ones, both with long-range correlations. We consider also a finite segment of natural DNA as part of the human chromosome $\mathrm{Ch} 22$. A more realistic approach was taken, compared with previous work, considering that the FermiDirac distribution was taken into account. Opposite to previous works, no harmonic oscillatory profile occurs in the temperature regions considered. Also, the qualitative form of the specific heat and chemical potential do not depend on the type and the size of the sequence used to model the DNA molecule. In particular, the slope of the chemical potential for high temperature depends on the number of band fillings $N_{e} / N$ disregarding the quasiperiodic sequence (see Figs. 1(b), 2(b) and 3(b)). Certainly the theoretical predictions shown in this Letter can be tested experimentally, and we expect that experimentalists get encouraged to face them.

\section{Acknowledgements}

This work was partially financed by the Brazilian Research Agency CNPq (projects CT-ENERG 554889/2006-4 and CNPq-Rede NanoBioestruturas 555183/2005-0).

\section{References}

[1] E.L. Albuquerque, M.G. Cottam, Phys. Rep. 376 (2003) 225.

[2] E. Maciá, Rep. Prog. Phys. 69 (2006) 397.

[3] S. Roche, Phys. Rev. Lett. 91 (2003) 108101.

[4] E.L. Albuquerque, M.S. Vasconcelos, M.L. Lyra, F.A.B.F. de Moura, Phys. Rev. E 71 (2005) 21910

[5] S. Karlin, V. Brendel, Science 259 (1993) 677.

[6] A. Rosas, E. Nogueira Jr., J.F. Fontanari, Phys. Rev. E 66 (2002) 61906.

[7] A.-M. Guo, Phys. Rev. E 75 (2007) 061915.

[8] F.D. Lewis, et al., Nature 406 (2000) 51.

[9] T. Terao, Phys. Rev. B 56 (1997) 975. 
[10] F.A.B.F. de Moura, M.L. Lyra, E.L. Albuquerque, J. Phys.: Condens. Matter 20 (2008) 075109.

[11] G. Gumbs, G.S. Dubey, A. Salman, B.S. Mahmoud, D. Huang, Phys. Rev. B 52 (1995) 210.

[12] P.W. Mauriz, M.S. Vasconcelos, E.L. Albuquerque, Physica A 294 (2001) 403; P.W. Mauriz, M.S. Vasconcelos, E.L. Albuquerque, Physica A 329 (2003) 101.

[13] H.Q. Yuan, U. Grimm, P. Repetowicz, M. Schreiber, Phys. Rev. B 62 (2000) 15569.

[14] C. Tsallis, L.R. da Silva, R.S. Mendes, R.O. Vallejos, A.M. Mariz, Phys. Rev. E 56 (1997) R4922.

[15] R.O. Vallejos, R.S. Mendes, L.R. da Silva, C. Tsallis, Phys. Rev. E 58 (1998) 1346

[16] L.R. da Silva, R.O. Vallejos, C. Tsallis, R.S. Mendes, S. Roux, Phys. Rev. E 64 (2001) 11104.
[17] D.J.B. Soares, M.L. Lyra, L.R. da Silva, Phys. Lett. A 318 (2003) 452.

[18] P.W. Mauriz, E.L. Albuquerque, M.S. Vasconcelos, Phys. Rev. B 63 (2001) 184203.

[19] H. Sugiyama, I. Saito, J. Am. Chem. Soc. 118 (1996) 7063.

[20] A.A. Voityuk, et al., J. Chem. Phys. 114 (2001) 5614.

[21] H. Zhang, et al., J. Chem. Phys. 117 (2002) 4578

[22] D.A. Moreira, E.L. Albuquerque, P.W. Mauriz, M.S. Vasconcelos, Physica A 371 (2006) 441.

[23] D.A. Moreira, E.L. Albuquerque, C.G. Bezerra, Eur. Phys. J. B 54 (2006) 393.

[24] P. Carpena, A.V. Coronado, P. Bernaola-Galván, Phys. Rev. E 61 (2000) 2281

[25] A.V. Coronado, P. Carpena, Phys. Rev. E 73 (2006) 016124.

[26] P. Carpena, P. Bernaola-Galván, A.V. Coronado, M. Hackenberg, J.L. Oliver, Phys Rev. E 75 (2007) 032903. 